Cómo citar este artículo: Hernández-Gil, C., \& Jaramillo-Gaitán, F. A. (2020). Laboratorio de innovación social: hibridación creativa entre las necesidades sociales y las experiencias significativas de los estudiantes de administración de empresas. Rev.investig.desarro.innov., 10 (2), 267-281.

\title{
Laboratorio de innovación social: hibridación creativa entre las necesidades sociales y las experiencias significativas de los estudiantes de administración de empresas
}

\section{Laboratory of social innovation: creative hybridization between the social needs and significant experiences of students of business administration}

\author{
Cristian Hernández-Gil' \\ Fabio Andrés Jaramillo-Gaitán² \\ Recibido: junio 25 de 2019 \\ Aceptado: diciembre 04 de 2019
}

\section{Resumen}

Se realizó, en el Programa de Administración de Empresas de la Universidad de la Amazonía, una investigación de corte cualitativo, con estudiantes de último semestre. El objetivo fue la aplicación de un laboratorio de innovación social, en el curso de ética empresarial, para fomentar la hibridación creativa entre las necesidades sociales de la ciudad de Florencia y los aprendizajes significativos de los estudiantes. El diseño metodológico utilizado fue la investigación-acción, en el cual participaron setenta estudiantes, elegidos a través de un muestreo no probabilístico. Los resultados arrojados fueron la invención de diferentes prototipos que los sujetos crearon a partir de la problemática planteada en el laboratorio. La mayoría de estos artefactos simbolizaron la fuerza o el poder como principal elemento para sensibilizar por el respeto a la vida. Se recomienda seguir desarrollando estas estrategias formativas, para promover en los futuros profesionales del saber administrativo, habilidades que solucionen dificultades no solo empresariales sino sociales, reforzando el trabajo en equipo, la creatividad y la capacidad para innovar.

Palabras clave: innovación social, ética empresarial, laboratorio, formación, respeto a la vida.

\begin{abstract}
A qualitative investigation was carried out in the Business Administration Program of the University of the Amazonia, with students from the last semester. The objective was the application of a laboratory of social innovation, in the course of business ethics, to promote creative hybridization between the social needs of the city of Florencia and the significant learning of students. The methodological design used was action research in which seventy students participated who were chosen through non-probabilistic sampling. The results obtained were the invention of different prototypes that the subjects created from the problem posed in the laboratory. Most of these artifacts symbolized strength or power as the main element to raise awareness about respect for life. It is recommended to continue developing these training strategies, to promote in future professionals of administrative knowledge, skills that solve not only business but social difficulties, reinforcing teamwork, creativity and the ability to innovate.
\end{abstract}

Keywords: social innovation, business ethics, laboratories, formation, respect for life.

1 Administrador de Empresas, Magíster en Ciencias de la Educación, Universidad de la Amazonía, Florencia, Colombia. E-mail: cris.hernandez@ udla.edu.co. ORCID: 0000-0001-6512-2453

2 Estudiante de Administración de Empresas, Universidad de la Amazonía, Florencia, Colombia. E-mail: jaramillogaitan@hotmail.com ORCID: 0000-0003-4916-712X 


\section{Introducción}

La ética sigue siendo una de las asignaturas pendientes en todos los programas de pregrado y posgrado de cualquier universidad ya sea pública o privada. Así lo evidencia el contexto actual, en el cual se insiste por la demanda de profesionales en el área empresarial que focalicen sus esfuerzos al alcance de la competitividad a través de una sola racionalidad, la técnica, la cual se fundamenta en la eficacia y la eficiencia de los procesos, dejando a un lado la responsabilidad social y la ética de cada actuación de estos sujetos (Guillén-Parra, 2006).

Cada día es preocupante el aumento del número de hechos que ponen en tela de juicio la integridad, la moralidad, y la dignidad del ser humano. Situaciones de corrupción, conflictos por intereses personales, así como algunas manipulaciones ideológicas, que promueven el odio, el rencor y demás sentimientos negativos que hacen que los ciudadanos se enfoquen en su individualidad y no en el bien común, reforzando aún más la vigencia de la frase célebre de Maquiavelo: "el fin justifica los medios". Por ejemplo, según Transparencia Internacional (2018), la percepción de corrupción en el sector público en Colombia está en aumento. El país cae de 37 a 36 puntos sobre 100 (la escala va de 0 a 100, siendo 0 "muy corrupto" y 100 "muy limpio") y desciende del puesto 96 al 99 entre 180 países cubiertos por la medición.

Desde esta perspectiva, y observando que, día a día, en ciudades como Florencia, capital del departamento del Caquetá, se presenta mayor número de homicidios por cuestiones de delincuencia común, el flagelo de la pobreza, así como la poca tolerancia ocasionada en los núcleos familiares, se hace necesario tomar medidas que permitan sensibilizar a las nuevas generaciones sobre esta problemática. Esto es especialmente cierto en los profesionales del saber administrativo, a quienes se debe enseñar sobre la importancia de la ética como elemento fundamental para el éxito laboral, personal, e incluso espiritual, a través de la formación en las aulas de clase.
Los homicidios en Colombia, aumentaron un 3,2 por ciento en el año 2018, pasando de 12.066 en 2017 a 12.458. También aumentó la tasa de homicidios por cada cien mil habitantes (de 27,4 y no de 25,8 como lo establece el Plan Nacional de Desarrollo). Esta situación ha tenido como causa principal el hurto en sus diferentes modalidades, especialmente a mano armada. Para el caso de la ciudad de Florencia, estos se redujeron en un $0,6 \%$ en el año 2017 (1691 casos) al año 2018 (1681 casos) (Acero-Velásquez, 2019).

En el Programa de Administración de Empresas de la Universidad de la Amazonía, se plantea una problemática, postulada por el sector productivo regional, relacionada con la poca capacidad de los egresados, en el diseño, creación e implementación de nuevos productos. Actualmente, el programa no cuenta con un conjunto de asignaturas que subsanen este vacío que tienen los profesionales en negocios, según los empresarios de la región. Es decir, no hay un curso, al menos, relacionado con la educación en innovación.

Es por ello que, articulando las dos problemáticas mencionadas, se propuso desde la asignatura de ética empresarial, aplicar laboratorios de innovación social. Estos se han convertido en una tendencia utilizada por organismos no gubernamentales, para que a través de la inventiva y la creatividad de la ciudadanía, se planteen necesidades y soluciones con miras a perfeccionar el planteamiento y ejecución de los planes de desarrollo territorial. En este caso, se busca relacionar los aprendizajes significativos de los sujetos de la investigación con las necesidades sociales de Florencia.

Como profesionales en administración de empresas, los estudiantes deben reconocer su realidad actual, analizándola e interpretándola, con el fin de desarrollar competencias propositivas, que impliquen el otorgamiento de soluciones a las necesidades y dificultades del entorno en el que viven y en el cual se desarrollarán profesionalmente. Se busca, a través del fomento 
de habilidades gerenciales y de responsabilidad social, que cada estudiante se asuma como integrante de la ciudadanía y aporte sus conocimientos y propuestas en favor de una causa, la cual, para esta investigación, enfatiza en el respeto a la vida. De esta forma, se busca dar respuesta al siguiente cuestionamiento: ¿Cómo se puede desarrollar en los estudiantes de administración de empresas de último semestre, cualidades de sensibilidad y responsabilidad social y ética frente a la vida, a partir de la creación de nuevos productos?

\section{Marco teórico y metodología}

\subsection{Referentes teóricos}

Los laboratorios de innovación se han convertido en lugares enmarcados en acciones de reflexión y trabajo mancomunado, que pertenecen a la categoría de innovación por pares o abierta (Fressoli, Arond, Abrol, Smith, Ely \& Días, 2014). El mundo, intervenido por las tecnologías digitales, requiere humanizar los procesos pedagógicos a través de estos espacios, dándole prioridad a la función social relacionada con la construcción de nuevos conocimientos de manera colaborativa, articulando el fin de la educación con el reconocimiento del desarrollo humano y económico. En los procesos educativos actuales, se ha dado apertura a los laboratorios sociales que buscan aplicar la innovación como solución a problemas de diferentes índoles. Los resultados de estos escenarios de aprendizaje, se desarrollan a partir de una pedagogía de pares (Rheingold, 2014), la cual es un insumo importante para el establecimiento de nuevas prácticas escolares que favorecen en los estudiantes, el perfeccionamiento de su análisis crítico y su pensamiento creativo.

Chesbrough (2003), habla de la innovación abierta como una proposición hacia un cambio de modelo económico, transformando las sinergias entre el sector público y la industria privada. El autor reconoce que el desarrollo solo se da cuando se pone a disposición de la ciudadanía la oportunidad de integrar los pensamientos de cada miembro en beneficio de un bien común.

Por su parte, Bordignon (2017), reconoce que, desde siempre los entes territoriales, en cada una de sus administraciones, han otorgado reconocimiento a la ciudadanía a través de la declaración de espacios públicos, como: centros de cultura, museos, bibliotecas, polideportivos, parques interactivos, entre otros. En estos, solo se recibe un servicio para una satisfacción individual y no integral. Con el cambio de paradigma sobre los mecanismos para generar desarrollo en las regiones, el autor explica que se concibe una modificación en las propuestas de espacios comunitarios para la acción social. Estos se enmarcan en instituciones llamadas, de manera genérica, laboratorios ciudadanos, que ofrecen plataformas que facilitan la participación de las personas en los procesos de experimentación y desarrollo de proyectos surgidos desde el territorio mismo. Es aquí donde se ejemplifica el modelo de la innovación abierta.

En estos laboratorios, cada persona o participante, ejerce su derecho hacia el saber hacer, con base a necesidades $u$ oportunidades que ve dentro del ejercicio público de sus libertades. Entonces, no se trata de generar nuevos conocimientos para ser transmitidos, sino en realizar acciones ciudadanas que puedan dar soluciones a problemas que los afectan directa o indirectamente. Según Hassan (2014), los laboratorios sociales emergen como plataformas clave para la experimentación social, en donde la colectividad y el sentido sistémico cobra validez, lo que los convierte en una herramienta educativa innovadora, que promueve a su vez en el alumnado un espíritu de indagación, colaboración y propensión al cambio social (Hillgren, Seravalli \& Emilson, 2011).

Marina (2010), abre el debate hacia la resignificación de la innovación educativa como una novena competencia esencial dentro del marco europeo de competencias claves para el aprendizaje permanente. Esta se denomina filosofía y ética, y busca apelar a una docencia que recupere el 
dinamismo propio del ser humano y estimule en el alumno la búsqueda de lo nuevo, para favorecer la creación y ampliación de sus posibilidades.

La mayoría de los laboratorios articulan el ejercicio de las funciones esenciales de los gobiernos, el sector privado y la academia. Esto les permite reestructurar cada elemento a priorizar dentro de los planes de desarrollo para darle una connotación holística a cada uno de ellos, y así cocrear políticas públicas efectivas (The Rockefeller Foundation, 2014).

En los laboratorios se desarrollan prácticas de colaboración a través de metodologías como el design thinking y la cocreación. Por ejemplo, el design thinking o pensamiento de diseño es una metodología basada en una serie de pasos como la empatía con el usuario, la definición del problema, el diseño de idea, la implementación de prototipos y el testeo (Steinbeck, 2011). En América Latina, todos los laboratorios, según un estudio reciente, han trabajado en conjunto con otros organismos del gobierno y con organismos no gubernamentales. De estos, el $82 \%$ han implementado herramientas de este tipo. En ciudades como Buenos Aires (Argentina), y Medellín (Colombia) con el programa Ciudad Inteligente, se perciben esfuerzos claros por extender redes con la comunidad de emprendedores para abordar problemas de la sociedad en los puntos de interacción entre el gobierno y los actores externos al mismo (Bordignon, 2017).

Para dar solución a problemáticas sociales, los equipos de personas que participan en los laboratorios de innovación social promueven prototipos sostenibles que pueden ser escalados tanto a nivel local como a nivel global. Las soluciones sociales que funcionan suelen ser imitadas y adaptadas, reutilizadas y replicadas ampliando su influencia hacia formar parte del mainstream de las soluciones sociales (Castro-Spila, Luna \& Unceta, 2016).

Roig-Vila (2017), realizó un estudio con empresas turísticas aplicando laboratorios ciudadanos. El encontró la necesidad de establecer relaciones sostenibles y compartir conocimientos tácticos, entre los usuarios y las empresas, para cocrear y desarrollar más y mejores productos y servicios. Gracias a este desarrollo, se comprobó la generación de competencias innovadoras de los estudiantes universitarios del área de turismo.

Una de las razones que justifican el desarrollo de esta investigación proviene del trabajo de Jurado-Pazy Morán-Vallejo (2019), quienes afirman que dentro de la población estudiantil existe un gran interés por participar en los programas sociales que se realizan en investigación e innovación social. Los autores reflexionan sobre la posibilidad, dentro de los claustros universitarios, del desarrollo de un modelo de gestión de la innovación que permita establecer una conexión de cooperación con el territorio. Esto facilitaría la interacción directa entre los estudiantes y la sociedad, permitiéndoles enfocar los conocimientos adquiridos de forma más práctica, interdisciplinar y transversal. Este modelo es una necesidad en la Universidad de la Amazonía, el cual podría funcionar a partir de la proyección social como camino principal que articule las exigencias del sector productivo, con la capacidad de los nuevos profesionales de abrirse a los requerimientos del entorno. Lo anterior es ratificado por Cruz-Rojas, Molina-Blandón y Valdiri-Vinasco (2019), quienes consideran que innovar en educación superior debe convertirse en un reto, que ponga en funcionamiento los elementos y sistemas que forman parte de la comunidad educativa.

Es así como, la innovación y el aprendizaje se encuentran vinculados fuertemente. La sociedad ha evolucionado a ser un conglomerado de la gestión del conocimiento, a través del trabajo colaborativo y en red de los diferentes actores institucionales que permiten consolidar procesos innovadores (Galeano-Barrera et al., 2018).

Los laboratorios de innovación social son espacios en donde integrantes de diferentes poblaciones, homogéneas o heterogéneas, presentan sus 
necesidades y las de sus comunidades para solucionarlas utilizando la creatividad y la innovación, a través de la construcción de prototipos con características de tangibilidad, promoviendo emprendimientos sociales o nuevos mecanismos para aplicarlos al desarrollo de las ciudades, poblaciones barriales, inclusive grupos familiares.

\subsection{Diseño metodológico}

El desarrollo metodológico se fundamentó en el enfoque cualitativo, que supone la evaluación de la mejora natural de los sucesos sin ningún tipo de manipulación o estimulación con respecto a la realidad (Hernández-Sampieri, Fernández-Collado \& Baptista-Lucio, 2015). Los sujetos de la investigación, estudiantes de últimos semestres del Programa de Administración de Empresas de la Universidad de la Amazonía, asumieron el reto de desarrollar una actividad pedagógica en el área de ética empresarial. En este caso, se indagó sobre la forma en la que ellos percibieron un problema en específico y sobre esa misma línea las distintas formas en las que le dieron solución o se aproximaron a dicha intención, partiendo de su experiencia, su aprendizaje significativo y su conocimiento hasta el momento sobre el tema, haciendo uso de la innovación.

El enfoque cualitativo se cimentó en una perspectiva interpretativa, centrada en el entendimiento del significado de las acciones propuestas por la unidad de análisis (los estudiantes), a través de sus prototipos. Dicha interpretación se define a partir del significado que le dan los participantes a la realidad del problema. De este modo, convergen varias "realidades", por lo menos la de los participantes, la de los investigadores y la que se produce mediante la interacción de todos los actores, las cuales también se modifican conforme transcurre el estudio y son la fuente de datos (Mercado-Martínez, 2002).

El diseño utilizado fue la investigación-acción participativa, el cual construye el conocimiento a través de un proceso práctico que involucra a los miembros del grupo o comunidad en todo el proceso de estudio y la implementación de acciones, producto de la indagación. En este caso, los resultados de la investigación se establecieron de las acciones realizadas por los participantes al momento de desarrollar el laboratorio, usando la técnica de Pensamiento de diseño (o design thinking), la cual da las pautas para la generación de nuevas innovaciones a partir de una problemática en específico, de orden social.

La investigación-acción inició con un proceso de construcción del fenómeno. Los estudiantes mediante su realidad imperante analizaron la problemática e incursionaron en diferentes escenarios para comprobar si esta ya había sido solucionada. Luego, al ver que no había una alternativa explícita que diera por hecho la erradicación de la situación problema, iniciaron un proceso creativo para plantear su propia solución (con la técnica pensamiento de diseño). Finalmente, pusieron a prueba su habilidad para trabajar en equipo y prototiparon la solución para luego sustentarla y validarla ante los demás grupos.

Los sujetos de la investigación fueron elegidos a través de un muestreo no probabilístico de tipo juicio. Los investigadores seleccionaron la unidad de análisis de acuerdo a los siguientes criterios: el participante debía tener matrícula vigente en el segundo periodo de 2018, en el Programa de Administración de Empresas; estar matriculado en la asignatura de ética empresarial; tener disposición y voluntad para ser parte de la investigación;y se requirió, que cada sujeto contara con la aprobación del setenta y cinco por ciento de los créditos académicos del programa. La unidad de trabajo se estructuró con un total de 70 estudiantes, tanto de la jornada nocturna como diurna, los cuales en equipos de cinco participantes conformaron catorce grupos para el desarrollo del laboratorio.

La técnica de recolección de datos fue la observación y el pensamiento de diseño. Esta última, impregna todo el espectro de actividades de innovación que se centra en el espíritu de diseño del 
hombre (Brown, 2008). Como enfoque, esta técnica no solo se centra en la creación de productos y servicios, sino también en la capacidad para que las personas sean intuitivas, reconozcan patrones, y construyan ideas con significado emocional y funcional (Brown, 2010).

El pensamiento de diseño implicó las siguientes 5 fases: En la primera, los equipos de trabajos se identificaron a través de un nombre que los representaría durante todo el laboratorio. Así mismo, cada integrante hizo su presentación personal y entre todos socializaron la siguiente problemática: ¿Cómo sensibilizar a un grupo social específico de la ciudad de Florencia, ubicada en el departamento del Caquetá, por el respeto a la vida individual y de los demás?

En la segunda fase, los equipos eligieron una población homogénea demográficamente y recibieron de los investigadores la explicación para la construcción de un mapa de dolor, en donde diagnosticaron la problemática situada desde los imaginarios y realidades del grupo social elegido, evaluando sus evidencias, relevancias, causas, entornos y perfiles. Al finalizar, cada equipo planteó una pregunta reto cuya leyenda fue: ¿Cómo podemos ayudar a (se escribe la población elegida) a que se sensibilice por el respeto a la vida propia y a la de los demás?
En la tercera fase, cada equipo indagó a través de fuentes secundarias (revisión documental), el estado de la cuestión o del arte de las posibles soluciones en diferentes contextos (internacional, nacional y regional) que se le han dado al problema planteado.

En la cuarta fase, cada integrante de cada equipo reflexionó, diseñó y planteó una propuesta de solución para la pregunta reto. Cada solución propuesta debería tener las siguientes características: ser concebida como un objeto tangible; y la solución (el prototipo o artefacto), además, debería ejecutar, de forma manual, las funciones suficientes para resolver el problema. En la parte final de esta fase, cada equipo construyó la solución (prototipo a escala usando materiales reciclados) integrando las ideas de todos los participantes.

En la última fase, los equipos validaron la solución, sustentándola frente a los demás. El alcance de la investigación fue el diseño del prototipo y el análisis de las características tangibles, patrones y símbolos que se representaron para darle solución a la problemática planteada. En la Figura 1 se aprecia el bosquejo general de las fases realizadas para el desarrollo del Laboratorio de Innovación Social.

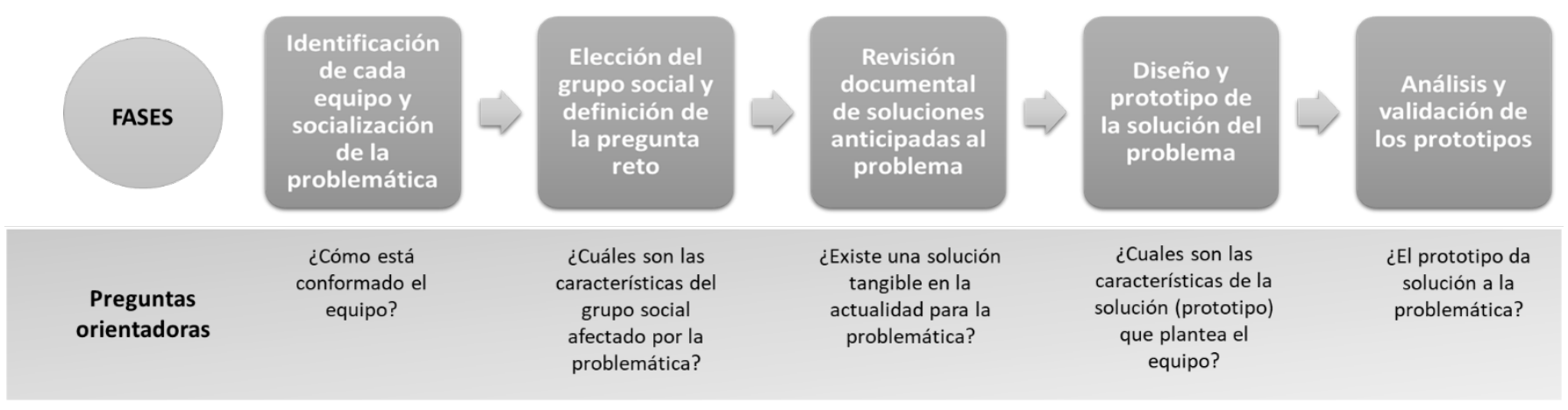

Figura 1. Fases del laboratorio de innovación social. 


\section{Resultados y discusión}

Los resultados se presentan de acuerdo al cumplimiento de las fases de la Figura 1. Cada equipo se identificó a través de un nombre característico o insignia. Así mismo, eligieron a un grupo social de la ciudad de Florencia, en el cual se podría presentar problemas de irrespeto por la vida y lo perfilaron a través del Mapa de Dolor, con seis variables: situación problema; perfil de la población afectada; evidencias a síntomas del problema; raíces o causas del problema; escenarios futuros en caso de que el problema persista; y factores del entorno que intervienen en la situación.

Los criterios establecidos por los equipos para elegir la población del problema fueron: i) el grupo social tiene algún tipo de relación con el entorno personal, social o familiar de los integrantes de cada equipo; ii) el grupo social fue elegido en consonancia con las noticias de las cuales han sido protagonistas en los últimos meses en el contexto local, relacionados con asesinatos y hurtos, y iii) el grupo social se identifica con las actitudes y acciones de los estudiantes universitarios. La elección de la población tuvo como generalidad el conjunto de personas que son agraviadas a través del hurto de sus pertenencias. Así mismo, resulta importante destacar que estos públicos se dedican a actividades remuneradas desde la informalidad, como, por ejemplo, los mototaxistas y los vendedores ambulantes.

En la Tabla 1, se presentan los problemas identificados a través de la pregunta reto y el perfil de cada grupo social seleccionado por los catorce equipos, dando cumplimiento, así, a las dos primeras fases.

Tabla 1. Resultado de las dos primeras fases del laboratorio.

\begin{tabular}{|c|c|c|c|}
\hline No. & $\begin{array}{l}\text { Nombre del } \\
\text { grupo }\end{array}$ & $\begin{array}{c}\text { Grupo social al cual va dirigido el } \\
\text { problema }\end{array}$ & Formulación del reto o problema \\
\hline 1 & $\begin{array}{l}\text { Los que } \\
\text { somos } 2.0\end{array}$ & $\begin{array}{l}\text { Estudiantes que no tienen transporte fijo } \\
\text { y al regresar a sus residencias luego de salir } \\
\text { de la universidad son víctimas de hurtos de } \\
\text { sus pertenencias especialmente el celular y } \\
\text { computador o dinero. }\end{array}$ & $\begin{array}{l}\text { ¿Cómo podemos ayudar a estudiantes } \\
\text { víctimas de hurtos de celulares a hacer } \\
\text { respetar su vida? }\end{array}$ \\
\hline 2 & $\begin{array}{c}\text { Súper } \\
\text { empleadores }\end{array}$ & $\begin{array}{l}\text { Profesionales o bachilleres de estrato uno o } \\
\text { dos que no poseen influencias políticas para } \\
\text { ubicarse laboralmente. }\end{array}$ & $\begin{array}{l}\text { ¿Cómo podemos ayudar a los desem- } \\
\text { pleados a que encuentren soluciones a } \\
\text { sus necesidades sin afectar la vida de los } \\
\text { demás? }\end{array}$ \\
\hline 3 & $\begin{array}{l}\text { Mundo } \\
\text { innovación }\end{array}$ & $\begin{array}{l}\text { Menores de edad de ambos sexos que sufren } \\
\text { de maltrato y violación en su entorno familiar } \\
\text { y social. }\end{array}$ & $\begin{array}{l}\text { ¿Cómo podemos ayudar a los niños a que } \\
\text { respeten su vida? }\end{array}$ \\
\hline 4 & $\begin{array}{l}\text { Comunidad } \\
\text { Jake }\end{array}$ & $\begin{array}{l}\text { Personas con enfermedades incurables y con } \\
\text { un nivel de motivación bajo y un marco de } \\
\text { depresión severo. }\end{array}$ & $\begin{array}{l}\text { ¿Cómo podemos ayudar a pacientes con } \\
\text { enfermedades terminales a que respeten } \\
\text { su propia vida? }\end{array}$ \\
\hline 5 & Hades & $\begin{array}{l}\text { Conductores con un nivel de inteligencia vial } \\
\text { bajo. }\end{array}$ & $\begin{array}{l}\text { ¿Cómo podemos ayudar a los conduc- } \\
\text { tores a que respeten la vida de los } \\
\text { peatones? }\end{array}$ \\
\hline 6 & Los increíbles & $\begin{array}{l}\text { Personas que roban a mano armada o con } \\
\text { herramientas blancas especialmente a estu- } \\
\text { diantes universitarios. }\end{array}$ & $\begin{array}{l}\text { ¿Cómo podemos ayudar a las personas } \\
\text { que hurtan celulares a que respeten la } \\
\text { vida de su víctima? }\end{array}$ \\
\hline 7 & $\begin{array}{l}\text { Salvadores } \\
\text { de } \\
\text { esperanzas }\end{array}$ & $\begin{array}{l}\text { Personas provenientes de comunidades del } \\
\text { campo en donde existen líderes sociales. }\end{array}$ & $\begin{array}{l}\text { ¿Cómo podemos ayudar a la comunidad } \\
\text { rural a que velen por la vida de sus líderes } \\
\text { sociales? }\end{array}$ \\
\hline
\end{tabular}




\begin{tabular}{|c|c|c|c|}
\hline No. & $\begin{array}{c}\text { Nombre del } \\
\text { grupo }\end{array}$ & $\begin{array}{c}\text { Grupo social al cual va dirigido el } \\
\text { problema }\end{array}$ & Formulación del reto o problema \\
\hline 8 & $\begin{array}{l}\text { Los chicos } \\
\text { del poder }\end{array}$ & $\begin{array}{l}\text { Mujeres menores de edad que inician su vida } \\
\text { sexual a los } 13 \text { y } 14 \text { años y resultan embara- } \\
\text { zadas sin ni siquiera haber terminado sus } \\
\text { estudios de secundaria. }\end{array}$ & $\begin{array}{l}\text { ¿Cómo podemos ayudar a las estudiantes } \\
\text { embarazadas a que respeten la vida de la } \\
\text { persona que se gesta en su vientre? }\end{array}$ \\
\hline 9 & $\begin{array}{l}\text { Los cinco } \\
\text { fantásticos }\end{array}$ & $\begin{array}{l}\text { Menores de edad de ambos sexos que sufren } \\
\text { de maltrato y violación en su entorno familiar } \\
\text { y social. }\end{array}$ & $\begin{array}{l}\text { ¿Cómo podemos ayudar a los niños y } \\
\text { niñas a que se protejan a sí mismos ante } \\
\text { una violación sexual? }\end{array}$ \\
\hline 10 & Los pilatos & $\begin{array}{l}\text { Personas de la ciudad de Florencia que } \\
\text { trabajan prestando el servicio de mototaxi. }\end{array}$ & $\begin{array}{l}\text { ¿Cómo podemos ayudar al gremio de los } \\
\text { Mototaxistas a que respeten su vida y la } \\
\text { de los demás? }\end{array}$ \\
\hline 11 & $\begin{array}{l}\text { Justicia por } \\
\text { las propias } \\
\text { manos }\end{array}$ & $\begin{array}{l}\text { Grupo de personas que hacen justicia con sus } \\
\text { propias manos a sujetos que interrumpen la } \\
\text { paz del barrio, hurtando casas o pertenencias } \\
\text { de los vecinos. }\end{array}$ & $\begin{array}{l}\text { ¿Cómo podemos ayudar a los vecinos } \\
\text { que toman la justicia por las manos a que } \\
\text { respeten la vida ajena? }\end{array}$ \\
\hline 12 & TANZ & $\begin{array}{l}\text { Personas caracterizadas por ser un ejemplo a } \\
\text { seguir en sus comunidades }\end{array}$ & $\begin{array}{l}\text { ¿Cómo podemos ayudar a los líderes } \\
\text { sociales a que hagan respetar su vida? }\end{array}$ \\
\hline 13 & Los agentes & $\begin{array}{l}\text { Agentes de policía que abusan del poder que } \\
\text { les confiere su oficio para agredir y atentar } \\
\text { contra la vida de otros. }\end{array}$ & $\begin{array}{l}\text { ¿Cómo podemos ayudar a los policías a } \\
\text { que respeten la vida de los vendedores } \\
\text { ambulantes? }\end{array}$ \\
\hline 14 & $\begin{array}{l}\text { Corporación } \\
\text { humanística }\end{array}$ & $\begin{array}{l}\text { Personas consumidoras de estupefacientes } \\
\text { que cometen hurtos para poder obtener } \\
\text { dinero para comprar su vicio. }\end{array}$ & $\begin{array}{l}\text { ¿Cómo podemos ayudar a los consumi- } \\
\text { dores de estupefacientes a que respeten } \\
\text { su propia vida? }\end{array}$ \\
\hline
\end{tabular}

En la tercera fase, el consenso de los equipos coincidió en la inexistencia, a la fecha, de una alternativa que sensibilizara el respeto a la vida a través de un artefacto o elemento tangible. Lo que más se aproximó a este objetivo, fue la aplicación legislativa desde los principios constitucionales (el uso del poder y el juzgamiento moral y civil), y el desarrollo de campañas publicitarias, de orden público, relacionadas con la generación de conciencia en contra del aborto y el suicidio. Lo más preocupante es que a nivel local no se han considerado soluciones a este flagelo.

En la fase cuarta se diseñó y construyó cada prototipo usando elementos del entorno (reciclaje). En conjunto, los catorce prototipos fueron analizados desde sus características tangibles y simbólicas. Este proceso se realizó con el software Atlas Ti. En la Figura 2, se presentan las características de tangibilidad y simbólicas utilizadas por los equipos para la elaboración de los artefactos. 


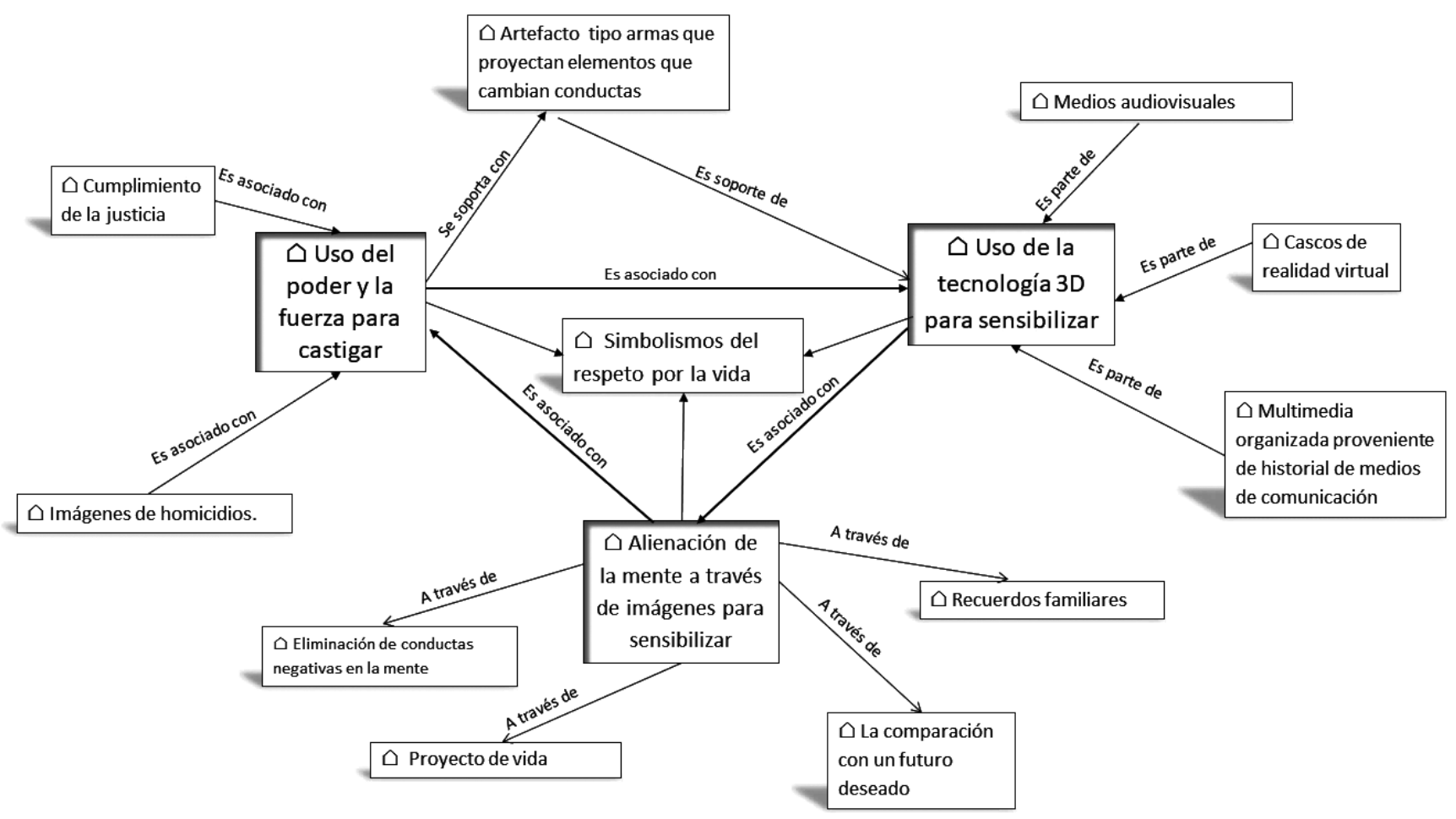

Figura 2. Red semántica con las características relevantes de los prototipos.

Es importante aclarar que los prototipos fueron construidos desde las capacidades empíricas y significativas que tienen los estudiantes, provenientes de formaciones recibidas durante su trayectoria educativa. Para los investigadores primó el consenso de todas las propuestas presentadas por los equipos y no un análisis individual de cada una, lo cual sería poco pertinente y aislada de los objetivos del estudio.

La primera característica exteriorizada en las propuestas innovadoras presentadas por los equipos, fue la utilización del poder y la fuerza para sensibilizar hacia el respeto a la vida. Es así como artefactos con forma de armas, o instrumentos de señalamiento como báculos y pistolas fumigadoras, buscaban dentro de sus funciones de sensibilización arrojar a los victimarios, balas o aromas, para un cambio de comportamiento o de mentalidad en dicho sujeto. Por ejemplo, el artefacto "Pistola Jake" tiene como función principal arrojar balas que al impactar al paciente con una enfermedad terminal (que intenta ponerle fin a su vida) permitan un cambio en su estado de ánimo reduciendo su cuadro depresivo. Otro ejemplo es una "bomba fumigadora" que, según su equipo creador, es capaz de esparcir aromas que trasmiten emociones como el amor y la humildad, a un sujeto al momento de cometer un delito.

En otros casos, dicho poder o fuerza para sensibilizar repercute en el cumplimiento de la ley. Según algunos equipos, los artefactos deben sensibilizar haciendo justicia de acuerdo a los contenidos en los códigos penal y civil, como única forma de hacer que una persona respete la vida. Por ejemplo, el dispositivo "scanner 2.0", se concibió como un estuche protector para celulares cuya función es contener un escáner de huellas dactilares, que al momento en el que el ladrón lo hurte, el escáner envía en tiempo real las huellas del delincuente al correo de la víctima para tener pruebas ante las autoridades. 
Otra característica representativa en los prototipos, tuvo que ver con la capacidad del artefacto para alienar la mente de la persona a la cual se quiere sensibilizar. Algunos prototipos hicieron uso de la realidad virtual y la multimedia. Por ejemplo, "Changing reality" fue diseñado como casco de realidad aumentada, cuya propuesta es que contenga tres niveles de complejidad para un cambio de mentalidad, de acuerdo a las características del sujeto a sensibilizar. Otro ejemplo es la "Cabina de la muerte", que consiste en un espacio para una sola persona, equipado con tecnología 4D E-motion, que cuenta con una silla con movimientos sincronizados y una pantalla interactiva con imágenes sugestivas y violentas de la forma como asesinan a líderes sociales.

En el desarrollo de las fases del pensamiento de diseño, la más compleja para los equipos fue la del diseño y prototipado. Expresiones como: "esto sí que lo pone a pensar a uno" y "no es posible hacerlo", fueron mencionadas varias veces dentro del laboratorio. La mayoría de los equipos inicialmente sufrieron un colapso o crisis hacia la acción durante el desarrollo del prototipo. No sabían cómo articular el problema con la necesidad del sujeto, y con la invención de un artefacto para su solución. En este punto se utilizó la hibridación como técnica de creatividad, la cual consiste en relacionar dos elementos, pensamientos o cosas, para crear un nuevo producto con funciones mixtas, proveniente de los objetos creadores.

Los resultados plantean un punto de partida que debe ser analizado dentro de la investigación y es la concepción de los estudiantes frente al concepto de innovación. Se plantean dos maneras de reconocer la percepción del hecho de innovar. Primero, como la inclusión tecnológica compleja a soluciones de la vida diaria, que va de la mano con sistemas de multimedia y componentes electrónicos que aseguran la novedad en cada invento. Los estudiantes de Administración de Empresas de la Universidad de la Amazonía, admiten que la innovación es para ingenieros. No están de acuerdo con que esa deba ser una función de un gerente, empresario o emprendedor. Por ello, reconocen las múltiples dificultades que presentaron en el desarrollo del laboratorio, al no tener los conocimientos técnicos, inclusive para hacer funcionar un bombillo o generar movimiento a través de una palanca.

La segunda manera es concebir la innovación como un proceso utópico que incluye elementos mágicos o surrealistas, como aromas que cambian las emociones y semillas que generan conciencia hacia la vida. En este sentido, no se tiene una clara definición de innovación, la cual, desde la literatura administrativa, se relaciona principalmente con la orientación hacia el mercado. Es decir, la introducción de nuevos valores, bienes o servicios para satisfacer a un grupo de consumidores (Damanpour \& Gopalakrishnan, 2001).

Otro aspecto a considerar, es el concepto de los estudiantes sobre el respeto a la vida. Se encuentran similitudes respecto a la percepción mostrada por los equipos a través de sus prototipos. SilveiraGorski (2000), explica que el sentido por la vida ha sufrido un proceso de cambios en su significación, sobre todo de orden social, manifestado en la educación orientada por la familia, los grupos y clases sociales, inclusive en contextos ideológicos y nacionalistas. En las propuestas presentadas, se vio el reflejo de diferentes perspectivas, a pesar de que el común denominador era orientar el artefacto a la sensibilización del victimario. Se evidenciaron los diferentes significados que los estudiantes le dieron a la vida, como: el respeto por la integridad y dignidad de los niños, la relevancia de los líderes sociales, la motivación que debe tener el enfermo terminal e inclusive los límites que debe tener una futura madre por la vida que engendra.

El concepto de vida es reconocido desde la prevención de múltiples situaciones, como conflictos, violencia, y consumo de drogas. Scales y Leffert (1999), a esto le suman la importancia de construir bienestar. Las personas, sobre todo los jóvenes, al experimentar un mayor nivel de bienestar personal, 
configurado en un sentimiento de ser competentes y sentir el apoyo de otros, tienen menos probabilidad de desarrollar comportamiento de riesgo, permitiendo así el mantenimiento de una buena salud, cuidado propio y acciones resilientes. Esto se evidenció en la mayoría de los prototipos, como en el "Magic Reality", que busca proyectar en la mente del delincuente, el futuro de su familia y la reacción de la misma al saber que es prófugo de la justicia por cometer una violación.

Pacheco-Zerga (2007), presenta la dignidad de la persona como un juicio que emite valor sobre la estructura específica del ser humano. Esta se encuentra relacionada con la capacidad de libertad y conocimiento del sujeto, lo que confirma que este valor pertenece a todos los individuos de la familia humana, sin discriminación alguna. No obstante, considera que los sucesos históricos han demostrado lo contrario, donde el respeto por la dignidad ha sido limitado en beneficio de los más fuertes. Esto queda demostrado en aquellos artefactos que buscan sensibilizar a la fuerza a los victimarios, hasta el punto de borrarles sus recuerdos negativos para que eviten perjudicar a los otros.

Así mismo, existe una teoría denominada elección racional, que explica como los infractores o delincuentes toman la decisión de cometer un atentado contra la integridad o la vida de una persona o grupo, basándose en la comparación de medir si el riesgo de ser descubiertos es superior o no, frente a los beneficios que se esperan lograr (Vozmediano-Sanz \& San Juan-Guillén, 2010). Esta situación se evidencia en el prototipo de "Changing reality", que muestra diferentes escenarios que anticipan el futuro del infractor, para que este tome conciencia.

La teoría del triángulo de la criminalidad (Cohen \& Felson, 1979, citados por Clarke \& Eck, 2008), afirma que el siniestro (hurto, asesinato), se origina cuando un infractor potencial y una víctima como objetivo concuerdan en espacio y tiempo, sin la presencia de una autoridad de la justicia y el orden. En este caso, de los catorce prototipos solo dos se desarrollan en coordinación con la actuación de las autoridades; estos son el "Brazalete de sensores" y la "Manilla 001", dos dispositivos útiles para favorecer la integridad de los menores de edad.

El último aspecto a resaltar en esta discusión, es el nivel de creatividad de los estudiantes para fortalecer sus competencias propositivas de innovación, principal reto de los futuros profesionales en los escenarios empresariales. La habilidad de crear va ligada a la necesidad de cambiar paradigmas (Ávalos-Dávila \& Sevillano-García, 2018). Los estudiantes se enfrentan a retos diarios en sus vidas, que los hacen cuestionar sobre la importancia de la creatividad para solucionar sus inconvenientes personales, familiares y/o sociales. Es allí donde las universidades deben reforzar el desarrollo competitivo del sujeto, en cada área del conocimiento. Así lo exponen Álvarez-Santullano y Prada-Creo (2018), quienes aseguran que la universidad tiene como papel esencial, el transmitir a sus alumnos, además de ciencia, valores como la creatividad, la iniciativa, la innovación, la responsabilidad, aspectos que van de la mano con el potencial emprendedor de los estudiantes.

Lo anterior justifica el uso de los laboratorios de innovación en las aulas de clase. Un estudiante que asume el reto de solucionar una problemática, puede desarrollar aprendizajes significativos que le aportan a la construcción de su proyecto de vida empresarial. En este sentido, se requiere de un trabajo continuo para el alcance del pensamiento divergente en contextos reales, en donde cada sujeto hace camino para lograr la profesionalidad. La creatividad se fundamenta en el principio de la interdisciplinariedad: es decir, dejar a un lado el aislamiento de los programas académicos, generando estrategias de articulación para promover soluciones integrales a problemáticas regionales, de orden social o económico. Difícilmente se podrán generar procesos de innovación en el quehacer profesional si no se amplía el campo de acción, a través de prácticas novedosas con 
otras disciplinas, como el método Lean Startup (Ávalos-Dávila \& Sevillano-García, 2018).

Se hace un llamado a los docentes del área del saber administrativo a hacer un alto en el camino y reflexionar sobre las didácticas utilizadas para la formación de administradores de empresas. Ya bien lo establecieron Osorio-Atehortúa y Malavera-Pineda (2018), quienes comprobaron las necesidades de darle un nuevo sentido a la enseñanza en todas las áreas de administración, más allá de las clases magistrales. Cada estudiante de negocios debe estar en la capacidad de generar aprendizajes significativos, a través de una formación creativa, constructiva y emotiva, haciendo uso, por ejemplo, de las lúdicas empresariales.

Igualmente, Oseda-Gago et al., (2019), postulan nuevas necesidades dentro de la formación, como lo es el planteamiento de fracasos en los resultados de cada actividad educativa. No siempre en los laboratorios de innovación habrá invenciones exitosas, ya que estas deberán pasar por un proceso de validación técnica y de mercados. Pero más allá de lograr la viabilidad de un prototipo, los estudiantes estarán en la capacidad de pensar y crear nuevos emprendimientos (Acevedo \& Dassen, 2016).

Investigaciones recientes demuestran la relación de proporcionalidad directa entre la capacidad para emprender y la capacidad para crear. Chávez (2015), expresa que ambas son innatas del ser humano y por ello es menester consolidar esfuerzos para garantizar la responsabilidad social, en los entes que conforman la dinámica empresarial. Finalmente, vale la pena dejar abierta esta discusión con lo expuesto por Romero-Haddad y De la Puente (2019), frente a la actuación de los profesionales de administración y su capacidad para ser sensibilizados en temas éticos, relacionados con los negocios y las problemáticas medioambientales. El sujeto en formación, a través de los laboratorios de innovación, estará en la capacidad de buscar y estudiar nuevas formas de gestión para responder a cuestiones sociales sin resolver.

\section{Conclusiones}

Las conclusiones se centran en tres aspectos: la indagación sobre posibles soluciones actuales a la problemática planteada en el laboratorio, en contextos regionales, nacionales e internacionales. En segundo lugar, la descripción de las características tangibles y simbólicas de los artefactos creados por los equipos $y$, en tercer lugar, la validación de estos frente al grupo de estudiantes.

En los contextos analizados por los estudiantes a través de la revisión documental, no se encontraron aportes significativos de otros autores o instituciones para solucionar la problemática del irrespeto a la vida. Lo que más se aproxima como alternativa, son las campañas hechas por gobiernos nacionales contra el aborto, el suicidio, el ciberbullying y la trata de blancas. Aun así, no se han elaborado artefactos tangibles que apoyen esta causa.

Los estudiantes diseñaron catorce prototipos a escala que permitían solucionar la problemática planteada o la pregunta reto. En general, todos los artefactos se caracterizaban por definir dos aspectos puntuales: la inventiva hacia el cuestionamiento de las normas y la utilización de la fuerza como único elemento que sensibiliza hacia el respeto a la vida. Los equipos priorizaron en la solución, las funciones utópicas que abarcan un proceso emancipador de las leyes. Se postulan alternativas de sensibilización que se centran más en el aspecto manipulador, a través de esquemas visuales y auditivos, que hacia el conocimiento y aplicación de la norma. Los equipos también identificaron que la única manera de sensibilizar a la comunidad, es utilizando herramientas que representan poder, incorporando así un enfoque autocrático del liderazgo.

En la validación de los prototipos, los estudiantes de administración sugirieron una segunda etapa 
del laboratorio o una sesión ampliada, donde se invitara a estudiantes del programa de Ingeniería de Sistemas. Esto para que hicieran segundos prototipos con realidad mecánica, técnica y electrónica, usando plataformas de hardware como Arduino y otros elementos de programación, manteniendo la versión original de los dispositivos del primer laboratorio.

Los esquemas de cocreación entre el laboratorio y las instituciones son necesarios para la construcción de un significado compartido alrededor de las innovaciones, permitiendo que haya sentimientos de apropiación por parte de los actores involucrados, lo cual ayudará a eliminar el paradigma de que "los administradores no innovan", así como el famoso "yo soy malo para ser creativo".

\section{Referencias}

Acero-Velásquez, H. (01 de abril de 2019). Más homicidios y más hurtos: la realidad de las ciudades colombianas. Diario Razón Pública. Bogotá, Colombia.

Acevedo, S., \& Dassen, N. (2016). Innovation for better management: The contribution of public innovation labs. Inter-American Development Bank. Recuperado de: https://publications.iadb. org/en/innovation-better-management-contribution-public-innovation-labs

Álvarez-Santullano, M. M., \& Prada-Creo, E. (2018). Evaluación de las competencias profesionales a través de las prácticas externas: incidencia de la creatividad. Revista de Investigación Educativa, 36 (1), 203-219. doi: http://dx.doi.org/10.6018/ rie.36.1.275651

Ávalos-Dávila, C., \& Sevillano-García, M. L. (2018). El desarrollo de competencias investigativas en la formación de estudiantes de la UNED de Costa Rica mediante la metodología Lean Startup. Revista Educatio Siglo XXI, 36 (3), 417-442. doi: https://doi. org $/ 10.6018 / \mathrm{j} / 350071$
Bautista, G., Borges, F., \& Forés, A. (2006). Didáctica universitaria en entornos virtuales de enseñanza-aprendizaje. Madrid, España: Narcea.

Bordignon, F. R. A. (2017). Laboratorios de innovación ciudadana, espacios para el hacer digital crítico. Virtualidad, Educación y Ciencia, 14 (8), 165-181. Recuperado de: http://sedici. unlp.edu.ar/bitstream/handle/10915/60952/ Documento_completo__.pdf-PDFA. pdf? sequence $=1 \&$ isAllowed $=y$

Brown, T., \& Wyatt, J. (2010). Design Thinking for Social Innovation. Stanford Social Innovation Review. 12 (1). 29 - 43. doi: https://doi. org/10.1596/1020-797X_12_1_29

Brown, T. (2008). Design thinking. Harvard business review, 86 (6), 1-10. Recuperado de: https://fusesocial.ca/wp-content/uploads/sites/2/2018/06/ Design-Thinking.pdf

Castro-Spila, J., Luna, A., \& Unceta, A. (2016). Social innovation regimes: an exploratory framework to measure social innovation. SIMPACT Working Paper No. 1, Institute for Work and Technology, 1-24. Gelsenkirchen: Alemania.

Chávez, A. (2015). Socialización de las competencias profesionales en varones y mujeres y sus implicaciones. Electronic Journal of Research in Educational Psychology, 7 (21), 466-474.

Chesbrough, H. W. (2003). Open Innovation: The New Imperative for Creating and Profiting from Technology. Boston, USA: Harvard Business School Press

Clarke, R. V., \& Eck, J. E. (2008). 60 pasos para ser un analista delictivo (1a.ed.). México: INACIPE.

Cohen, L., E., \& Felson, M. (1979). Social Change and Crime Rate Trends: A Routine Activity Approach. American Sociological Review, 44 (4), 588-608. Recuperado de: www.jstor.org/stable/2094589 
Cornish, D. B., \& Clarke, R. V. (2008). The Rational Choice Perspective. Wortley, R., \& Mazerolle, Y. Environmental Criminology and Crime Analysis. Portland, Oregón: Willan.

Cruz-Rojas, G., Molina-Blandón, M., \& Valdiri-Vinasco, V. (2019). Vigilancia tecnológica para la innovación educativa en el uso de bases de datos y plataformas de gestión de aprendizaje en la universidad del Valle, Colombia. Revista de Investigación, Desarrollo e Innovación, 9 (2), 303-317. doi: https:// doi.org/10.19053/20278306.v9.n2.2019.9175

Damanpour, F., \& Gopalakrishnan, S. (2001): The dynamics of the adoption of product and process innovations in organizations. Journal of Management Studies. 38 (1). 45-65.

Fressoli, M., Arond, E., Abrol, D., Smith, A., Ely, A., \& Días, R. (2014). When grassroots innovation movements encounter mainstream institutions: implications for models of inclusive innovation. Innovation and Development, 4 (2), 277-292. doi: https://doi.org/10.1080/2157930X.2014.921354

Galeano-Barrera, C., Bellón-Monsalve, D., Zabala-Vargas, S., Romero-Riaño, E., \& Duro-Novoa, V. (2018). Identificación de los pilares que direccionan a una institución universitaria hacia un smart-campus. Revista de Investigación, Desarrollo e Innovación, 9 (1), 127-145. doi: https://doi. org/10.19053/20278306.v9.n1.2018.8511

Guillen-Parra, M. (2006). Ética en las organizaciones: construyendo confianza. Pearson Prentice Hall.

Hassan, Z. (2014). The social labs revolution: A new approach to solving our most complex challenges. San Francisco, CA, USA: Berrett-Koehler.

Hernández-Sampieri., R. Fernández-Collado, C., \& Baptista-Lucio., P. (2015). Metodología de la Investigación. México, D.F.: McGraw-Hill.
Hillgren, P. A., Seravalli, A., \& Emilson, A. (2011). Prototyping and infrastructuring in design for social innovation. CoDesign, 7 (3-4), 169-183. doi: https://doi.org/10.1080/15710882.2011.630474

Jurado-Paz, I., \& Morán-Vallejo, M. (2019). Gestión universitaria de la innovación social promovida desde espacios académicos relacionados con el emprendimiento, la investigación y la proyección social. Revista de Investigación, Desarrollo e Innovación, 9 (2), 261-272. doi: https://doi. org/10.19053/20278306.v9.n2.2019.9161

Marina, J. A. (2010). La competencia de emprender. Revista de Educación, 351, 49-71. Recuperado de: https://goo.gl/qfrbaJ

Mercado-Martínez, F. J. (2002). Investigación cualitativa en América Latina: Perspectivas críticas en salud. International Journal of Qualitative Methods, 1 (1), 1-27. Recuperado de: https://sites.ualberta. $\mathrm{ca} /$ iiqm/backissues/1_1Final/pdf/mercadospanish.pdf

Oseda-Gago, D., Mendivel-Gerónimo, R. K., Oliva-Paredes, R., Vargas-Flores, J. I., \& Patiño-Rivera, A. R. (2019). Autoeficacia Emprendedora y Capacidad Creativa en la Universidad Nacional Intercultural de la Amazonía. Repositorio de Revistas de la Universidad Privada de Pucallpa, 3 (03), 6-11. Recuperado de: http://revistas.upp.edu. pe/index.php/RICCVA/article/view/114

Osorio-Atehortúa, U. A., \& Malavera-Pineda, S. M. (2018). Medios digitales emergentes en la implementación de didácticas específicas en el programa de administración de empresas. Prisma Social: revista de investigación social, (21), 438-457. Recuperado de: https://dialnet.unirioja.es/servlet/ articulo?codigo $=6521446$

Pacheco-Zerga, L. (2007). El "derecho de morir" y el "deber de matar" por respeto a la dignidad humana. Revista de Derecho Universidad de Piura. 
8 (8). 45-60. Recuperado de: https://pirhua.udep. edu.pe/handle/11042/2623

Rheingold, H. (2014). The Peeragogy Handbook. A guide for Peer-Learning and Peer Production, 2. Arlington, Estados Unidos: MA Peirce Press.

Roig-Vila, R. (2017). Investigación en docencia universitaria. Diseñando el futuro a partir de la innovación educativa. Barcelona, España: Ediciones Octaedro, S.L.

Romero-Haddad, C. M., \& De la Puente, K. I. (2019). Diagnóstico sobre la Prospectiva de Generación de Empresas y Proyectos Productivos de Emprendimiento Social y Economías Creativas en el Sector Solidario, Propuestos por Estudiantes de la UCC Sede Montería año 2017. Crescendo, 10 (1), 241 261. doi: https://doi.org/10.21895/incres.2019. v10n 1.14

Scales, P. C., \& Leffert, N. (1999). Developmental assets: $A$ synthesis of the scientific research on adolescent development. Minneapolis, MN: Search Institute.
Silveira-Gorski. H. C. (2000). La vida en común en sociedades multiculturales. Aportaciones para un debate. Identidades comunitarias y democracia, 11-46. Madrid, España: Trotta.

Steinbeck, R. (2011). "Building Creative Competence in Globally Distributed Courses through Design Thinking", Comunicar, 37 (19). 27-35. Recuperado de: http://eprints.rclis.org/16746/1/ en27-35.pdf

The Rockefeller Foundation. (2014). "Social Innovation Labs. How Social Innovation Labs Can Advance your Work." Recuperado de: http://globalknowledgeinitiative.org/pdf/Sociallnnovation-Labs-External-Guide.pdf

Transparencia Internacional. (2018). Índice de Percepción de Corrupción (IPC). Recuperado de: http://transparenciacolombia.org.co/ informe-anual-2018/

Vozmediano-Sanz, L., \& San Juan-Guillén, C. (2010). Criminología ambiental. Ecología del delito y de la seguridad. España: Editorial UOC. 
